\title{
Effect of Sensorimotor Integration Exercise on Balance Among Patients with Stroke
}

\author{
Sang-hun Jang', Onseok Lee ${ }^{2}$ \\ ${ }^{1}$ Department of Physical Therapy, Gimcheon University \\ Samrak-dong, Gimcheon-si, Gyeongsangbuk-do, 740-704 Republic of Korea \\ upsh22@hanmail.net \\ ${ }^{2}$ Department of Medical IT Engineering, Soonchunhyang University \\ 22 Soonchunhyang-ro, Sinchang-myeon, Asan-si, Chungcheongnam-do, 31538 Republic of Korea \\ on-seok@hanmail.net
}

\section{Extended Abstract}

This study attempts to investigate the impact that the sensorimotor integration exercise has on the recovery of balance among patients with stroke by examining the muscle activity and limit of stability (LOS) [1] [2].

A total of 24 subjects participated. The subjects were randomly allocated by the computer program to one of two groups control (CON) group $(n=12)$, sensorimotor integration exercise (SIE) group $(n=12)$. The research subjects received intervention five days a week for a total of four weeks. CON group additionally received 30-minute general balance training, while SIE group additionally received 30-minute sensory integration training.

In the muscle activity, the improvement of Erector spinae (ES) and Gluteus medius (GM) was more significant in SIE group than CON group. In the LOS, the improvement of affect side and forward side was significantly higher in SIE group compared to CON group.

Sensorimotor integration exercise can improve balance ability of patients with stroke by increasing muscle activity of stance limb muscles such as GM and trunk extensor such as ES along with enhancement of the limit of stability [3].

\section{References}

[1] I. V. Bonan, F. M. Colle, J. P. Guichard, E. Vicaut, M. Eisenfisz, P. Tran Ba Huy and A. P. Yelnik, "Reliance on visual information after stroke. Part I: balance on dynamic posturography," Arch Phys Med Rehabil., vol. 85, no. 2, pp. 268-273, 2004.

[2] C. B. Oliveira, Í. R. T. Medeiros, M. G. Greters, N. A. F. Frota, L. T. Lucato, M. Scaff and A. B. Conforto, "Abnormal sensory integration affects balance control in hemiparetic patients within the first year after stroke," Clinics., vol. 66, no. 12, pp. 2043-2048, 2011.

[3] D. F. Graham, C. P. Carty, D. G. Lloyd, G. A. Lichtwark and R. S. Barrett, "Muscle contributions to recovery from forward loss of balance by stepping," J Biomech., vol. 47, no. 3, pp. 667-674, 2014. 